\title{
Características clínicas e efeitos do Covid-19 nos pacientes idosos: uma revisão integrativa
}

Clinical characteristics and effects of covid-19 in elderly patients: an integrative review

Características clínicas y efectos del covid-19 en pacientes ancianos: una revisión integradora

\author{
Victor Alves NASCIMENTO ${ }^{1}$ \\ Jovânia Alves OLIVEIRA ${ }^{1}$ \\ Mariana Nunes Godoi MOREIRA ${ }^{1}$ \\ Jader Bueno DE OLIVEIRA ${ }^{1}$ \\ Vinicius Rafael GONZAGA ${ }^{1}$ \\ Marcela Filié HADDAD ${ }^{2}$
}

${ }^{1}$ Aluno(a) do Curso de graduação em Odontologia, Faculdade de Odontologia, Universidade Federal de Alfenas (UNIFAL-MG), 37.130-000 Alfenas - MG, Brasil

${ }^{2}$ Cirurgiã-Dentista, Mestre e Doutora em Prótese, Professora da Disciplina de Prótese Total Removível da Faculdade de Odontologia,

Universidade Federal de Alfenas (UNIFAL-MG), 37.130-000 Alfenas - MG, Brasil

\section{Resumo}

Introdução: A COVID-19 tornou-se uma emergência de saúde pública no ano de 2020, sendo caracterizada como pandemia no mês de março. Os idosos compõem o grupo de risco dessa doença. Objetivo: Avaliar os efeitos da pandemia de COVID-19 sobre os idosos. Material e Método: A busca pelos artigos foi realizada através da plataforma Pubmed, com a inclusão de 17 estudos. Resultados: Os resultados ressaltaram a prevalência da doença entre a população idosa, e evidenciaram que esse grupo apresenta sintomas atípicos e com duração ligeiramente mais longa desde o início da sintomatologia até a sua admissão, tornando-se mais difícil a identificação da infecção de forma precoce. As manifestações clínicas mais relatadas foram: febre, tosse, produção de escarro, diarreia, fadiga, pneumonia e lesão cardíaca aguda. Também se observa que comorbidades, como hipertensão e doença pulmonar obstrutiva crônica, levam a uma disfunção cardíaca e pulmonar devido a mudanças fisiológicas e anatômicas dos pulmões, agravando o quadro clínico dos pacientes. Os exames de imagem se mostraram grandes aliados ao diagnóstico das alterações pulmonares decorrentes da infecção, além da realização do exame RT-PCR. Conclusão: Os idosos compõem o grupo de risco da COVID-19, sendo indivíduos com comorbidades os mais susceptíveis à agravamentos clínicos. Há necessidades em seguir as recomendações da OMS por não haver um tratamento específico destinado a essa patologia.

Descritores: Infecções por Coronavírus; Idoso; Diagnóstico Clínico.

\section{Abstract}

Introduction: COVID-19 became a public health emergency in 2020, and in March it was characterized as a pandemic. The elderly make up the risk group for this disease. Objective: To assess the effects of the COVID-19 pandemic on the elderly. Material and Method: The search for the articles was carried out through the Pubmed platform, with the inclusion of 17 studies. Results: The results highlighted the prevalence of the disease among the elderly population, and showed that this group has atypical symptoms and has a slightly longer duration from the beginning of the symptoms until their admission, making it more difficult to identify the infection early. The most reported clinical manifestations were: fever, cough, sputum production, diarrhea, fatigue, pneumonia and acute cardiac injury. It is also observed that comorbidities, such as hypertension and chronic obstructive pulmonary disease, lead to cardiac and pulmonary dysfunction due to physiological and anatomical changes in the lungs, worsening the clinical condition of patients. Imaging exams proved to be great allies to the diagnosis of pulmonary changes resulting from the infection, in addition to the RT-PCR exam. Conclusion: The elderly make up the COVID-19 risk group, with individuals with comorbidities being the most susceptible to clinical worsening. There is a need to follow WHO recommendations as there is no specific treatment for this pathology.

Descriptors: Coronavirus infection; Aged; Clinical Diagnosis.

\section{Resumen}

Introducción: COVID-19 se convirtió en una emergencia de salud pública en 2020, y en marzo se caracterizó como una pandemia. Los ancianos constituyen el grupo de riesgo para esta enfermedad. Objetivo: Evaluar los efectos de la pandemia COVID-19 en los ancianos. Material y método: La búsqueda de los artículos se realizó a través de la plataforma Pubmed, con la inclusión de 17 estudios. Resultados: Los resultados destacaron la prevalencia de la enfermedad entre la población anciana, y mostraron que este grupo presenta síntomas atípicos y tiene una duración algo mayor desde el inicio de los síntomas hasta su ingreso, lo que dificulta la identificación precoz de la infección. Las manifestaciones clínicas más reportadas fueron: fiebre, tos, producción de esputo, diarrea, fatiga, neumonía y daño cardíaco agudo. También se observa que comorbilidades, como la hipertensión y la enfermedad pulmonar obstructiva crónica, conducen a disfunción cardíaca y pulmonar debido a cambios fisiológicos y anatómicos en los pulmones, empeorando la condición clínica de los pacientes. Los exámenes de imagen demostraron ser grandes aliados para el diagnóstico de cambios pulmonares derivados de la infección, además del examen de RTPCR. Conclusión: Los ancianos constituyen el grupo de riesgo para COVID-19, siendo los individuos con comorbilidades los más susceptibles al empeoramiento clínico. Es necesario seguir las recomendaciones de la OMS ya que no existe un tratamiento específico para esta patología.

Descriptores: Infecciones por Coronavírus; Anciano; Diagnóstico clínico.

\section{INTRODUÇÃO}

No dia 31 de dezembro de 2019, a Organização Mundial da Saúde (OMS) foi alertada sobre diversos casos de pneumonia na cidade de Wuhan, província de Hubei, na China. Tratava-se de uma nova cepa de coronavírus que ainda não fora identificada antes em seres humanos. Em 30 de janeiro de 2020, o surto do vírus Sars-Cov-2 (Severe Acute Respiratory Syndrome Coronavirus) ou COVID-19 (Corona Virus Disease-19) constitui uma Emergência de Saúde Pública de importância Internacional, definida como o mais alto nível de alerta da OMS, e no dia 11 de março do mesmo ano, foi caracterizada como pandemia pela mesma instituição ${ }^{1}$.

De acordo com a Organização Pan Americana de Saúde (OPAS), as formas de contágio da COVID-19 compreendem desde o contato direto com pessoas infectadas (através de gotículas de saliva ou secreções respiratórias expelidas ao tossir, espirrar ou falar) ao contato através de superfícies inanimadas contaminadas. Ademais, alguns procedimentos médicos e odontológicos podem produzir aerossóis os quais ficam suspensos no ar e uma vez contaminados pelo vírus podem contaminar 
profissionais e pacientes no ambiente em questão ${ }^{2}$.

Segundo a OMS, foram confirmados no mundo todo até o dia 19 de novembro de 2020, 55 928327 casos de COVID-19 e 1344003 mortes. No Brasil, de acordo com o Ministério da Saúde, há 5.945.849 casos confirmados de coronavírus e 167,455 óbitos até o dia 19 de novembro de $2020^{1,3}$.

Ademais, dentre os grupos de riscos destacam-se os idosos, pessoas com comorbidades, as quais agravam o quadro clínico da doença. É importante dizer que os idosos com doenças préexistentes, como diabetes e hipertensão, apresentam maior taxa de mortalidade, soma-se a isso a senilidade que pode agudizar os sintomas da doença. Além disso, é visto que o uso de ventilação mecânica aumenta conforme a idade ${ }^{4,5}$.

Dessa forma, o objetivo do presente trabalho foi realizar uma revisão integrativa da literatura acerca dos efeitos da pandemia de COVID-19 sobre os idosos.

\section{MATERIAL E MÉTODO}

A busca foi realizada na plataforma Pubmed via MEDLINE, com os descritores: "COVID- 19" OR "Coronavirus disease 2019" OR "SARS-CoV-2 infection" OR "Coronavirus Infections" AND "Health of Institutionalized Elderly" OR "Elderly patients". A partir desta busca foram encontrados 81 artigos. Os critérios de exclusão foram: artigos não redigidos em língua inglesa, revisões de literatura, carta ao editor e pesquisas que não correlacionaram idosos e COVID-19. Assim, restaram 17 artigos para serem lidos na íntegra e comporem a presente revisão integrativa.

\section{RESULTADOS}

Os resultados da presente revisão integrativa se encontram dispostos na tabela 1. Todos os estudos incluídos nesta revisão abordaram a relação entre idosos e a COVID-19 são do ano de 2020. A amostra final foi constituída por 10 estudos retrospectivos, 3 estudos clínicos, 1 estudos descritivos, 2 estudos transversais e 1 estudo observacional. As principais considerações referentes ao tema em questão encontradoas nestes estudos são exibidos nas Tabelas 1 a 4.

\section{DISCUSSÃO}

De acordo com a revisão de literatura realizada, os estudos acerca da COVID-19 tendo como população alvo os idosos, analisaram as características epidemiológicas, manifestações clínicas da doença neste grupo; os exames de diagnóstico e acompanhamento da evolução da doença; o impacto de fatores sistêmicos (fatores de risco) no agravo do quadro; as formas de tratamentos e as orientações de acompanhamento.

A literatura avaliada apresentou características epidemiológicas dos indivíduos diagnosticados com COVID-19. A maioria dos acometidos são idosos e do sexo masculino. A taxa de mortalidade foi maior em pacientes acima de 80 anos. As doenças crônicas subjacentes aumentam as chances de mortalidade nesses pacientes ${ }^{6,7}$. Também foi observado que os grupos de alto risco devem ter proteção adequada contra infecções e acesso precoce a cuidados médicos quando infectados, para garantir a sobrevida desses pacientes ${ }^{8}$.

Tabela 1: Autor, ano, objetivos, tipo e principais considerações dos artigos incluídos no estudo
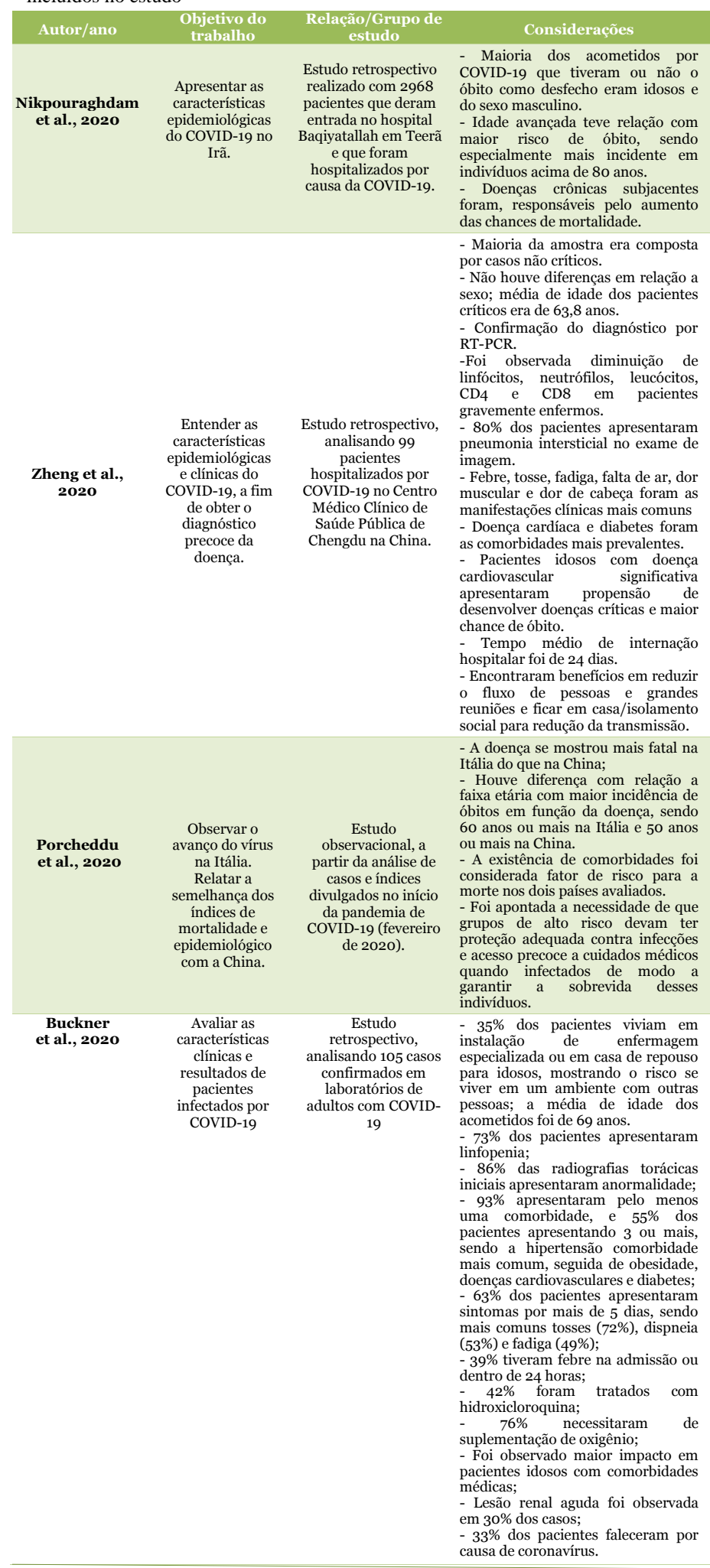

Estudo retrospectivo, analisando 99 hospitalizados po COVID-19 no Centro Médico Clínico de Saúde Publica de
Chengdu na China. precoce da

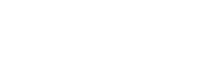
chance de óbito. Tempo médio de

Encontraram benefícios em reduzir fluxo de pessoas e grandes social para redução da transmissão. A doença se mostrou mais fatal na Houve diferença com relação a em função da doença, sendo anos ou mais na Itália e 50 anos A existência de comorbidades fo considerada fator de risco para Foi apontada a necessidade de que risco devam ter acesso precoce a cuidados médicos antir a sobrevida desses 35 dos pacientes viviam em de enfermagem para idosos, mostrando o risco se pessoas; a média de idade dos cometidos foi de 69 anos. $73 \%$ dos pacientes apresentaram $86 \%$ das radiografias torácica (ciais apresentaram anormalidade; ma comorbidade, e $55 \%$ dos pacientes apresentando 3 ou mais, nais comum, seguida de obesidade, $63 \%$ dos pacientes apresentaram intomas por mais de 5 dias, sendo mais comuns tosses ( $72 \%$ ), dispneia
(53\%) e fadiga ( $49 \%$ ); 9\% tiveram febre na admissão o entro de 24 horas; de Foi observado maior impacto em páéntes idosos com comorbidades Lesão renal a causa de coronavírus. 
Tabela 2: Autor, ano, objetivos, tipo e principais considerações dos artigos incluídos no estudo

\begin{tabular}{|c|c|c|c|}
\hline Autor/ano & $\begin{array}{l}\text { Objetivo do } \\
\text { trabalho }\end{array}$ & $\begin{array}{l}\text { Relação/Grupo de } \\
\text { estudo }\end{array}$ & Considerações \\
\hline $\begin{array}{l}\text { Liu Kui et al., } \\
2020\end{array}$ & $\begin{array}{l}\text { Relatar as } \\
\text { manifestações } \\
\text { clínicas, } \\
\text { resultados de } \\
\text { exames } \\
\text { laboratoriais, } \\
\text { características } \\
\text { das imagens e } \\
\text { protocolos/regi } \\
\text { me de } \\
\text { tratamento de } \\
\text { pacientes com } \\
\text { coronavírus. }\end{array}$ & $\begin{array}{c}\text { Estudo } \\
\text { retrospectivo, } \\
\text { observando } 137 \\
\text { pacientes de } 20 \text { a } 83 \\
\text { anos infectados pelo } \\
\text { novo coronavírus }\end{array}$ & $\begin{array}{l}\text { - Pessoas de meia-idade e idosos se } \\
\text { apresentaram mais suscetíveis a } \\
\text { infecção; o principal meio de } \\
\text { transmissão foi homem-homem. } \\
\text { - Maioria dos pacientes apresentou } \\
\text { contagem normal ou reduzida de } \\
\text { glóbulos brancos e mais de } 70 \% \\
\text { desenvolveram linfocitopenia. } \\
\text { - } 84,7 \% \text { das imagens pulmonares } \\
\text { mostraram acometimento bilateral; } \\
\text { - Pacientes gravemente enfermos } \\
\text { tiveram pior prognóstico devido a } \\
\text { comorbidade subjacente. } \\
\text { - Febre, tosse, mialgia ou fadiga } \\
\text { foram os sintomas mais comuns. } \\
\text { - Alguns pacientes de meia-idade e } \\
\text { idosos sem comorbidades tiveram } \\
\text { febre moderada, baixa ou inexistente } \\
\text { durante a doença; 20\% tiveram } \\
\text { disfunção de órgãos, principalmente } \\
\text { insuficiência renal. } \\
\text { - Pacientes com doença moderada } \\
\text { ou grave necessitam de } \\
\text { hospitalização; tratamentos foram } \\
\text { focados no suporte sintomático e } \\
\text { respiratório. } \\
\text { - Taxa de mortalidade por covid-19 } \\
\text { é menor que da SARS; período de } \\
\text { incubação pode ser maior. }\end{array}$ \\
\hline $\begin{array}{l}\text { Zhao et al., } \\
\quad 2020\end{array}$ & $\begin{array}{c}\text { Comparar as } \\
\text { diferenças } \\
\text { clínicas de } \\
\text { pacientes } \\
\text { diagnosticados } \\
\text { com COVID-19, } \\
\text { divididos em } \\
\text { grupos de } \\
\text { idades } \\
\text { diferentes. }\end{array}$ & $\begin{array}{l}\text { Estudo retrospectivo } \\
\text { realizado com } 1000 \\
\text { pacientes com } \\
\text { COVID-19 divididos } \\
\text { em três grupos de } \\
\text { diferentes idades:o } \\
\text { primeiro grupo } \\
\text { composto por } \\
\text { indivíduos com } \\
\text { idade inferior a } 60 \\
\text { anos; segundo grupo } \\
\text { entre } 60 \text { e } 74 \text { anos e } \\
\text { terceiro grupo com } \\
\text { idade igual ou } \\
\text { superior a } 75 \text { anos. }\end{array}$ & $\begin{array}{l}\text { - De acordo com o aumento da } \\
\text { idade, a contagem de glóbulos } \\
\text { brancos e neutrófilos aumentou, } \\
\text { enquanto a de linfócitos diminuiu. } \\
\text { - Das } 545 \text { tomografias, } 509 \\
\text { apresentaram pneumonia (230 com } \\
\text { idade superior a } 60 \text { anos). } \\
\text { - Idosos tiveram sintomas mais } \\
\text { atípicos (dificultando a identificação } \\
\text { precoce da infecção por COVID-19) e } \\
\text { mais comorbidades com danos nos } \\
\text { órgãos, disfuncão imunológica e } \\
\text { inflamação mais grave na admissão. } \\
\text { - Prognóstico ruim pode estar } \\
\text { associado a maior comorbidade em } \\
\text { pacientes idosos; sintomas comuns } \\
\text { entre todos os pacientes foram febre, } \\
\text { tosse, fadiga e dispneia. } \\
\text { - Taxa de febre em pacientes idosos } \\
\text { foi menor que em pacientes jovens. } \\
\text { - Com o aumento da idade, houve } \\
\text { maior incidência de dispneia e } \\
\text { menor taxa de dor muscular. } \\
\text { - A comorbidade mais comum foi } \\
\text { hipertensão, seguida de diabetes. } \\
\text { - Grupo mais velho apresentou mais } \\
\text { complicaçôes que o grupo jovem. } \\
\text { - 191 pacientes apresentaram } \\
\text { complicaçôes, sendo mais comum a } \\
\text { lesão cardíaca aguda (97 indivíduos } \\
\text { com idade acima de 6o anos). } \\
\text { - 6,3\% dos pacientes (63) foram } \\
\text { encaminhados para a UTI, sendo os } \\
\text { pacientes mais velhos mais } \\
\text { propensos a esta medida. } \\
\text { - 119 pacientes morreram, com } \\
\text { maior proporção para maior idade. }\end{array}$ \\
\hline $\begin{array}{l}\text { Wang et al., } \\
2020 .\end{array}$ & $\begin{array}{l}\text { Investigar as } \\
\text { características e } \\
\quad \text { fatores } \\
\text { prognósticos em } \\
\text { pacientes idosos } \\
\text { com COVID-19. }\end{array}$ & $\begin{array}{c}\text { Estudo clínico em } \\
\text { Idosos (+6o anos) } \\
\text { com COVID 19. }\end{array}$ & $\begin{array}{l}\text { Dispneia, linfocitopenia, } \\
\text { comorbidades incluindo doença } \\
\text { cardiovascular e doença pulmonar } \\
\text { obstrutiva crônica e síndrome do } \\
\text { desconforto respiratório agudo } \\
\text { foram preditores de mau resultado. } \\
\text { - Alta proporção de casos graves a } \\
\text { críticos e alta taxa de mortalidade } \\
\text { foram observadas nos pacientes } \\
\text { idosos com COVID-19. rigoroso e } \\
\text { Monitoramento rigo } \\
\text { tratamento oportuno devem ser } \\
\text { realizados para pacientes idosos de } \\
\text { alto risco. }\end{array}$ \\
\hline $\begin{array}{l}\text { Guo et al., } \\
2020\end{array}$ & $\begin{array}{l}\text { Analisar e } \\
\text { resumir as } \\
\text { características } \\
\text { clínicas de } \\
\text { pacientes idosos } \\
\text { com COVID-19. }\end{array}$ & $\begin{array}{l}\text { Estudo retrospectivo } \\
\text { para comparar as } \\
\text { diferenças entre } \\
\text { pacientes idosos } \\
\text { jovens (6o-74 anos) } \\
\text { e idosos ( } \geq 75 \text { anos). }\end{array}$ & 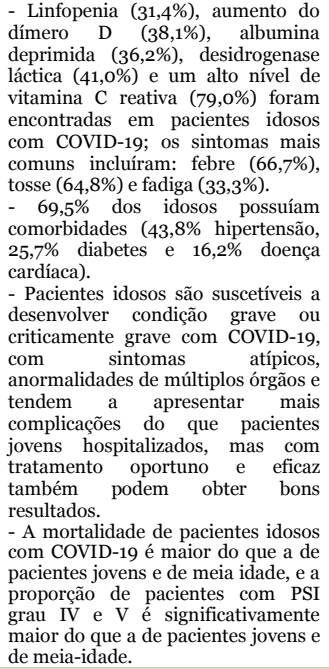 \\
\hline
\end{tabular}

Tabela 3: Autor, ano, objetivos, tipo e principais considerações dos artigos incluídos no estudo

\begin{tabular}{|c|c|c|c|}
\hline Autor/ano & $\begin{array}{l}\text { Objetivo do } \\
\text { trabalho }\end{array}$ & $\begin{array}{l}\text { Relação/Grupo de } \\
\text { estudo }\end{array}$ & Considerações \\
\hline $\begin{array}{l}\text { Liu Kai et al., } \\
2020\end{array}$ & $\begin{array}{l}\text { Analisar as } \\
\text { características } \\
\text { clínicas de } \\
\text { pacientes idosos } \\
\text { com pneumonia } \\
\text { por COVID-19 }\end{array}$ & $\begin{array}{c}\text { Estudo retrospectivo } \\
\text { com } 56 \text { pacientes } \\
\text { que apresentavam } \\
\text { pneumonia em } \\
\text { decorrência do } \\
\text { COVID- } 19 \\
\text { hospitalizados em } \\
\text { Hainan, de } 15 \text { de } \\
\text { janeiro a } 18 \text { de } \\
\text { fevereiro de } 2020 . \\
\text { Os pacientes } \\
\text { comparados foram } \\
\text { com idade maior ou } \\
\text { igual a } 60 \text { anos } \\
\text { (idosos) e menor do } \\
\text { que } 60 \text { anos (jovens } \\
\text { e meia-idade). }\end{array}$ & $\begin{array}{l}\text { - Um total de } 56 \text { pacientes foram } \\
\text { avaliados, sendo } 18 \text { idosos }(32,14 \%) \\
\text { e } 38 \text { jovens e de meia idade } \\
(67,86 \%) \text {. } \\
\text { - A proporção de linfócitos no grupo } \\
\text { de idosos foi significativamente } \\
\text { menor que a no grupo jovem e de } \\
\text { meia idade; e a proténa C reativa } \\
\text { foi significativamente maior no } \\
\text { grupo jovem. } \\
\text { - Os sintomas mais comuns em } \\
\text { ambos os grupos foram febre, } \\
\text { seguida de tosse e escarro. } \\
-0 \text { indice de gravidade de } \\
\text { pneumonia graus IV e V e a } \\
\text { proporção de envolvimento } \\
\text { múltiplo dos lobos foram maiores } \\
\text { nos idosos do que nos jovens e de } \\
\text { meia idade. } \\
\text { - Não houve diferença nas lesões de } \\
\text { lobo único entre os dois grupos. }\end{array}$ \\
\hline $\begin{array}{l}\text { Liu Kai et al., } \\
2020\end{array}$ & $\begin{array}{l}\text { Investigar os } \\
\text { efeitos do } \\
\text { treinamento de } \\
\text { reabilitação } \\
\text { respiratória de 6 } \\
\text { semanas na } \\
\text { função } \\
\text { respiratória, } \\
\text { qualidade de vida } \\
\text { (QV), mobilidade } \\
\text { e função } \\
\text { psicológica em } \\
\text { pacientes idosos } \\
\text { com COVID-19. }\end{array}$ & $\begin{array}{l}\text { Estudo clínico } \\
\text { randomizado com } \\
\text { Idosos com COVID- } \\
\text { 19, avaliando } \\
\text { parâmetros } \\
\text { respiratórios após } \\
\text { reabilitação. }\end{array}$ & $\begin{array}{l}\text { - A reabilitação respiratória de seis } \\
\text { semanas pode melhorar a função } \\
\text { respiratória, QV e ansiedade em } \\
\text { pacientes idosos com COVID-19, } \\
\text { mas não apresenta melhora } \\
\text { significativa no estado depressivo } \\
\text { do idoso e atividades do dia a dia. }\end{array}$ \\
\hline Li et al., 2020 & $\begin{array}{c}\text { Avaliar a } \\
\text { prevalência de } \\
\text { desnutrição e seus } \\
\text { fatores } \\
\text { relacionados em } \\
\text { pacientes idosos } \\
\text { com COVID-19 } \\
\text { em Wuhan, } \\
\text { China. }\end{array}$ & $\begin{array}{l}\text { Estudo transversal } \\
\text { com } 182 \text { pacientes } \\
\text { idosos internados } \\
\text { com COVID-19. }\end{array}$ & $\begin{array}{l}\text { - A prevalência de desnutrição em } \\
\text { pacientes idosos com COVID-19 foi } \\
\text { alta }(52,7 \%) \text {. } \\
\text { - O suporte nutricional deve ser } \\
\text { reforçado, especialmente para } \\
\text { aqueles com diabetes mellitus, com } \\
\text { baixa circunferência da panturrilha } \\
\text { ou baixa albumina. } \\
\text { - O mau prognóstico geral de } \\
\text { COVID-19 aos idosos pode estar } \\
\text { relacionado ao mau estado } \\
\text { nutricional dos mesmos. }\end{array}$ \\
\hline $\begin{array}{l}\text { Niu et al., } \\
2020\end{array}$ & $\begin{array}{l}\text { Analisar } \\
\text { características } \\
\text { clínicas de } \\
\text { pacientes idosos } \\
\text { com COVID-19. }\end{array}$ & $\begin{array}{l}\text { Estudo retrospectivo } \\
\text { para comparar } \\
\text { diferenças entre 50- } \\
64 \text { anos, } 65-79 \text { anos } \\
\text { e mais de } 80 \text { anos. }\end{array}$ & 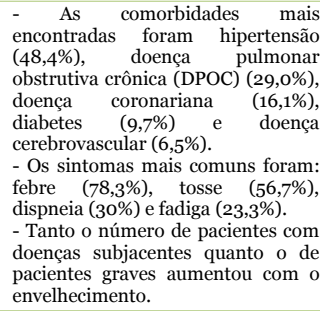 \\
\hline Lee et al., 2020 & $\begin{array}{c}\text { Elucidar as } \\
\text { características } \\
\text { clínicas e os } \\
\text { fatores de risco } \\
\text { para mortalidade } \\
\text { e necessidade de } \\
\text { ventilação } \\
\text { mecânica (VM) ou } \\
\text { cânula nasal de } \\
\text { alto fluxo (HFNC) } \\
\text { em pacientes } \\
\text { idosos } \\
\text { hospitalizados } \\
\text { com COVID-19. }\end{array}$ & $\begin{array}{l}\text { Estudo retrospectivo } \\
\text { envolvendo idosos } \\
\text { hospitalizados por } \\
\text { COVID-19. }\end{array}$ & $\begin{array}{l}\text { - Pacientes com idade } \geq 80 \text { anos } \\
\text { apresentaram alto risco de } \\
\text { necessidade de VM / HFNC. } \\
\text { A mortalidade entre os pacientes } \\
\text { com doença grave pré-existente foi } \\
\text { extremamente alta. } \\
\text { - Doenças pré-existentes comuns a } \\
\text { essa faixa etária são fatores } \\
\text { relacionados com a probabilidade } \\
\text { de um maior risco de morte nesse } \\
\text { grupo de pacientes por promoverem } \\
\text { o agravamento da doença } \\
\text { provocada pela COVID-19. }\end{array}$ \\
\hline $\begin{array}{c}\text { Fatyga et al., } \\
2020\end{array}$ & $\begin{array}{c}\text { Estudo sobre } \\
\text { orientação médica } \\
\text { por telefone, } \\
\text { durante a } \\
\text { pandemia de } \\
\text { COVID-19, em } \\
\text { pacientes idosos } \\
\text { com diabetes tipo 2 }\end{array}$ & $\begin{array}{c}\text { Estudo transversal } \\
\text { analisando } 86 \\
\text { pacientes com mais } \\
\text { de } 60 \text { anos. Utilizou- } \\
\text { se a telemedicina } \\
\text { para realizar o } \\
\text { controle glicêmico e } \\
\text { do estado emocional. }\end{array}$ & $\begin{array}{l}\text { - A telemedicina pode ajudar a } \\
\text { controlar e reduzir a ansiedade em } \\
\text { pacientes com diabetes tipo } 2 \\
\text { durante o surto de COVID-19. }\end{array}$ \\
\hline
\end{tabular}

Condições coexistentes mais comuns foram: hipertensão $(36,3 \%)$, diabetes $(17,6 \%)$, doença cardiovascular $(14,5 \%)$ e doença pulmona

Pacientes idosos com comorbidades precisam de mais cuidados médicos. - Os principais sintomas foram: febre $(78,9 \%)$, tosse ( $49 \%)$, dispneia (31,9\%), produçáo de escarro (18,1\%), angustia torácica $(16,2 \%)$, fadiga $(15,2 \%)$, anorexia $(15,2 \%)$ Dispneia, TnI e D-díme elevados admissão são fatores de risco para marta - Diagnóstico precoce e cuidados suporte são de grande importância para os

- Pacientes idosos do COVID-19 apresentam maior taxa de mortalidade. 
Tabela 4: Autor, ano, objetivos, tipo e principais considerações dos artigos incluídos no estudo

\begin{tabular}{|c|c|c|c|}
\hline Autor/ano & $\begin{array}{l}\text { Objetivo do } \\
\text { trabalho }\end{array}$ & $\begin{array}{l}\text { Relação/Grupo de } \\
\text { estudo }\end{array}$ & Considerações \\
\hline $\begin{array}{c}\text { Kumar et al., } \\
2020\end{array}$ & $\begin{array}{l}\text { Checar se a } \\
\text { suplementação } \\
\text { de Zn } \\
\text { demonstra um } \\
\text { potencial para a } \\
\text { profilaxia e } \\
\text { tratamento do } \\
\text { COVID-19 }\end{array}$ & $\begin{array}{c}\text { Estudo descritivo } \\
\text { baseado na análise } \\
\text { da literatura acerca } \\
\text { do potencial } \\
\text { benefício do zinco } \\
\text { para profilaxia do } \\
\text { tratamento do novo } \\
\text { coronavírus }\end{array}$ & $\begin{array}{l}\text { - Sugere- } \text { se que a } \\
\text { suplementação de Zn pode } \\
\text { beneficiar a profilaxia e e } \\
\text { tratamento do COVID-19, } \\
\text { devido à ausência de terapias } \\
\text { eficazes para esta doença }\end{array}$ \\
\hline $\begin{array}{l}\text { Ward et al., } \\
2020\end{array}$ & $\begin{array}{c}\text { Identificar o } \\
\text { estado mental } \\
\text { alterado (AMS) } \\
\text { como marcador } \\
\text { clínico do } \\
\text { COVID-19, } \\
\text { mesmo na } \\
\text { ausência de } \\
\text { problemas } \\
\text { respiratórios e } \\
\text { febre }\end{array}$ & $\begin{array}{c}\text { Estudo clínico a } \\
\text { partir do } \\
\text { acompanhamento de } \\
4 \text { idosos sem } \\
\text { problemas } \\
\text { respiratórios ou } \\
\text { febre que } \\
\text { apresentaram estado } \\
\text { mental alterado } \\
\text { devido à infeção por } \\
\text { COVID-19 }\end{array}$ & $\begin{array}{l}\text { - AMS pode ser um dos } \\
\text { primeiros sintomas clínicos da } \\
\text { infecção por COVID-19. } \\
\text { - Os idosos são os mais } \\
\text { vulneráveis às complicações } \\
\text { adversas do COVID e a AMS } \\
\text { deve ser considerada como um } \\
\text { possivel sinal de infecção para } \\
\text { iniciar o tratamento no início do } \\
\text { curso da doença, talvez antes } \\
\text { que os sintomas respiratórios } \\
\text { sejam aparentes. }\end{array}$ \\
\hline
\end{tabular}

Os estudos evidenciaram que a população idosa é mais susceptível à contaminação por COVID19, bem como à evolução de quadros graves. Além disso, demonstrou-se um amplo espectro clínico da doença nesta população, na qual foi constatada sintomatologia mais agressiva, diversa e relacionada com situações de saúde pré-existentes à infecção ${ }^{6,7,9-13}$. Observa-se que os idosos apresentam sintomas mais atípicos e com duração ligeiramente mais longa desde o início da sintomatologia até a sua admissão, tornando-se mais difícil a identificação da infecção por COVID-19 de forma precoce. Como, por exemplo, o estado mental alterado, demonstrado pelo estudo de Ward $^{14}$, que pode ser um dos primeiros sinais clínicos da infecção por COVID-19 e esse sinal deve ser considerado como um indicativo da doença, possibilitando o tratamento com antecedência, mesmo sem os sintomas respiratórios e febre $^{14}$. Além disso, uma questão que pode explicar a ocorrência de quadros graves é o fato de haver uma propagação da infecção devido à não percepção desses sintomas atípicos, contribuindo para o seu mau prognóstico ${ }^{11}$.

É visto na literatura a existência de pacientes assintomáticos, sendo que estes requerem a devida atenção, sendo necessário o rastreamento de seus contatos e o isolamento o mais rápido possível ${ }^{10}$. Porém, nota-se que muitos estudos se dedicaram a elucidar as manifestações clínicas mais frequentes, sendo estas: febre, tosse, produção de escarro, anorexia, diarreia, fadiga, mialgia, dor de cabeça, acometimento dos lobos pulmonares, pneumonia, disfunção de órgãos (principalmente insuficiência renal), lesão cardíaca aguda, dispnéia, diminuição de linfócitos, neutrófilos, leucócitos CD4 e CD8 em pacientes gravemente enfermos, depressão de albumina, desidrogenase láctica e alto nível de vitamina $\mathrm{C}$ reativa. A idade e a associação com comorbidades tornou a doença mais agressiva e a morte foi mencionada como desfecho para os casos mais graves ${ }^{4,5,7,8-13,15-17}$. Tais manifestações podem estar relacionadas a maior prevalência de comorbidades preexistentes nesses pacientes, como hipertensão arterial, diabetes mellitus e doenças cardiovasculares, tornando-os mais susceptíveis à SARS-CoV-2, uma vez que doenças metabólicas foram relatadas como causadoras de imunossupressão. Além disso, pode haver associação de um mau estado nutricional dos mesmos. De acordo com os estudos, se observa algumas disfunções ocorridas por comorbidades, como hipertensão e doença pulmonar obstrutiva crônica (DPOC) levando a uma disfunção cardíaca e pulmonar. Assim, podem se associar ao mau prognóstico, juntamente com os fatores que acompanham o aumento da idade, como mudanças na anatomia dos pulmões e atrofia de músculos, acarretando mudanças fisiológicas do sistema respiratório ${ }^{5,11,16}$

Para identificação laboratorial das alterações decorrentes da COVID-19, estudos mostraram que foram utilizados contagens sanguíneas padrão (avaliação de leucócitos absolutos e linfócitos), bioquímica sanguínea (verificando-se alanina transaminase, aspartato transaminase, creatina quinase, creatinina), função de coagulação, entre outros. Da mesma forma, demonstraram que os exames de imagem são grandes aliados para o diagnóstico de alterações pulmonares decorrentes da infecção, representadas por um aspecto pulmonar denso, opacidade em vidro fosco e coexistindo com consolidação ou sombras em forma de cordão. Por fim, o exame RT-PCR é utilizado para auxiliar no diagnóstico definitivo por meio de uma coleta de secreções do trato respiratório ${ }^{4,10,15}$. Portanto, a associação desses exames tem a possibilidade de acarretar um diagnóstico definitivo por COVID-19, visto que há relatos de que o vírus pode afetar os linfócitos T (células TCD4+), com um quadro de diminuição do número de linfócitos do paciente ${ }^{11}$.

A literatura aponta uma série de patologias como fatores sistêmicos agravantes ou fatores de risco para a COVID-19. Estas incluem diabetes, hipertensão, doenças cardiovasculares, doenças cerebrovasculares, DPOC, síndrome do desconforto respiratório agudo, alterações articulares degenerativas, desnutrição e obesidade ${ }^{9,12,16,18}$. Li et al. ${ }^{19}$ afirmam que a assistência médica é maior para os pacientes idosos com comorbidades prévias, além de uma maior taxa de internação em unidades de terapia intensiva. Isso se deve ao agravamento dessas condições após o contágio com a COVID-19 Uma possível explicação para o fato é a baixa proporção de linfócitos e o sistema imunológico debilitado desses pacientes quando comparados a paciente jovens e de meia-idade ${ }^{4,15,17,19}$. Achados encontrados no estudo de Porcheddu et al. ${ }^{8}$ corroboram para tanto, uma vez que cerca de 10 a 16 pacientes falecidos por COVID-19 apresentaram comorbidades préexistentes ${ }^{8}$. É digno de destacar que as doenças préexistentes (hipertensão, doença cardíaca coronária, DPOC, diabetes e doença cerebrovascular) em 
pacientes idosos são, em sua maioria, incapacitantes, como evidenciam Niu et al. ${ }^{17}$.

O controle do quadro clínico estabelecido pela COVID-19 é realizado através de acompanhamento e terapia medicamentosa. Fatyga et al. ${ }^{18}$ sugerem a telemedicina para controlar e reduzir a ansiedade em pacientes diabéticos durante a pandemia. Este acompanhamento se justifica pela limitação das consultas presenciais devido à pandemia, e sua praticabilidade, uma vez que é possível a realização de prescrições medicamentosas, diagnóstico, ações de prevenção e educação em saúde $^{18}$.

Uma forma de tratamento sugerida por $\mathrm{Liu}^{4}$ é a reabilitação respiratória, que consiste em intervenções de treinamento muscular respiratório, realizado com um dispositivo de resistência (Threshold PEP; Philips Co.); exercícios de tosse, treinamento diafragmático; alongamento dos músculos respiratórios e exercícios em casa conforme as instruções do terapeuta ${ }^{4}$. Ademais, Kumar et al. ${ }^{20}$ descrevem o uso da suplementação de zinco com o objetivo de aumentar a imunidade antiviral, tanto inata quanto humoral, e restaurar a função das células imunes esgotadas ou para melhorar a função das células imunes normais, em particular em pacientes imunocomprometidos ou idosos, sugerindo que essa suplementação pode ser benéfica para a profilaxia e tratamento da COVID-19.

\section{CONSIDERAÇÕES FINAIS}

Os resultados dessa revisão integrativa da literatura evidenciaram que a maior taxa de incidência e mortalidade da COVID-19 ocorre na população idosa. Além disso, quando associada a alguma comorbidade sistêmica (diabetes, hipertensão, doenças cardiovasculares, doenças cerebrovasculares, doença pulmonar obstrutiva crônica, síndrome do desconforto respiratório agudo, alterações articulares degenerativas, desnutrição e obesidade), a doença se torna mais agressiva e com a evolução de quadros mais graves, com um maior risco de morte. Vários possíveis tratamentos estão sendo estudados, porém, ainda não há nenhum com a eficácia comprovada. Também não há vacinas disponíveis contra a COVID-19 autorizadas pela OMS até o momento. Enquanto não existem tratamentos e devido ao maior risco dessa população idosa, se faz de extrema importância seguir as recomendações da OMS no que diz respeito à utilização de máscara de proteção; lavagem frequente das mãos com água e sabão ou, na impossibilidade desta, limpeza com álcool em gel; quando tossir ou espirrar cobrir a boca com o antebraço ou com um lenço descartável e manter ao menos 1 metro de distância das outras pessoas.

\section{AGRADECIMENTOS}

Esta pesquisa teve o fomento do Ministério de Educação e Cultura (MEC), através da concessão de bolsas do Programa de Educação Tutorial (PET) aos seus autores.

\section{REFERÊNCIAS}

1. World Health Organization [homepage internet]. Pandemia de doença por coronavirus (COVID-19) [acesso em 19 nov 2020]. Disponível em: http: https://www.who.int/emergencies/diseases/novelcoronavirus-2019?gclid=CjwKCAiAzN j9BRBDEiwAPsLOdMtvP29HNfG_fKThMRyfZF5ZAhTrrwKXEDh7 4AOZIY7ddRjJ_su0 hoCK3EQAvD_BwE.

2. Organização Pan-Americana de Saúde [homepage na internet]. Folha informativa COVID-19 Escritório da OPAS e da OMS no Brasil [acesso em: 19 nov. 2020]. Disponível em: https://www.paho.org/pt/covid19.

3. Ministério da Saúde [homepage na internet]. Painel Coronavirus Brasil [acesso em: 19 nov. 2020]. Disponível em: http:https://covid.saude.gov.br/.

4. Liu K, Zhang W, Yang Y, Zhang J, Li Y, Chen Y. Respiratory rehabilitation in elderly patients with COVID-19: A randomized controlled study. Complement The Clin Pract. 2020:101166.

5. Lee JY, Kim HA, Huh K, Hyun M, Rhee J-Y, Jang S, et al. Risk Factors for Mortality and Respiratory Support in Elderly Patients Hospitalized with COVID-19 in Korea. J Korean Med Sci. 2020;35(23)

6. Nikpouraghdam M, Farahani AJ, Alishiri G, Heydari S, Ebrahimnia M, Samadinia H, et al. Epidemiological characteristics of coronavirus disease 2019 (COVID-19) patients in IRAN: A single center study. J Clin Virol. 2020; 127:104378.

7. Zheng Y, Xu H, Yang M, Zeng Y, Chen H, Liu R, et al. Epidemiological characteristics and clinical features of 32 critical and 67 noncritical cases of COVID-19 in Chengdu. J Clin Virol. 2020; 127:104366.

8. Porcheddu R, Serra C, Kelvin D, Kelvin N, Rubino S. Similarity in case fatality rates (CFR) of COVID-19/SARS-COV-2 in Italy and China. J Infect Dev Ctries. 2020;14(02):125-8.

9. Buckner FS, McCulloch DJ, Atluri V, Blain M, McGuffin SA, Nalla AK, et al. Clinical Features and Outcomes of 105 Hospitalized patients with COVID-19 in Seattle, Washington. Clin Infect Dis. 2020;71(16):2167-73.

10. Liu K, Fang Y-Y, Deng Y, Liu W, Wang M-F, Ma J-P, et al. Clinical characteristics of novel coronavirus cases in tertiary hospitals in Hubei Province. Chin Med J. 2020;133(9):1025-31. 
11. Zhao M, Wang M, Zhang J, Gu J, Zhang P, Xu Y, et al. Comparison of clinical characteristics and outcomes of patients with coronavirus disease 2019 at different ages. Aging (Albany NY). 2020;12(11): 10070.

12. Wang L, He W, Yu X, Hu D, Bao M, Liu H, et al. Coronavirus disease 2019 in elderly patients: Characteristics and prognostic factors based on 4week follow-up. J Infect. 2020;80(6):639-45.

13. Guo T, Shen Q, Guo W, He W, Li J, Zhang Y, et al. Clinical Characteristics of Elderly Patients with COVID-19 in Hunan Province, China: A Multicenter, Retrospective Study. Gerontol. 2020:1-9.

14. Ward CF, Figiel GS, McDonald WM. Altered Mental Status as a Novel Initial Clinical Presentation for COVID-19 Infection in the Elderly. Am J Geriatr Psychiatry. 2020; 28(8):808-11.

15. Liu K, Chen Y, Lin R, Han K. Clinical features of COVID-19 in elderly patients: A comparison with young and middle-aged patients. $\mathrm{J}$ Infect. 2020;80(6):e-14-8.

16. Li T, Zhang Y, Gong C, Wang J, Liu B, Shi L, et al. Prevalence of malnutrition and analysis of related factors in elderly patients with COVID-19 in Wuhan, China. Eur J Clin Nutr. 2020:1-5.

17. Niu S, Tian S, Lou J, Kang X, Zhang L, Lian H, et al. Clinical characteristics of older patients infected with COVID-19: A descriptive study. Arch Gerontol Geriatr. 2020;89:104058.

18. Fatyga E, Dzięgielewska-Gęsiak S, Wierzgoń A, Stołtny D, Muc-Wierzgon M. The coronavirus disease 2019 pandemic: telemedicine in elderly patients with type 2 diabetes. Pol Arch int Med. 2020;130(5):452-54.

19. Li P, Chen L, Liu Z, Pan J, Zhou D, Wang H, et al. Clinical Features and Short-term Outcomes of Elderly Patients With COVID-19. Int J Infect Dis. 2020;97:245-50.

20. Kumar A, Kubota Y, Chernov M, Kasuya H. Potential Role of Zinc Supplementation in Prophylaxis and Treatment of COVID-19. Med Hypotheses. 2020;144:109848.

\section{CONFLITO DE INTERESSES}

Os autores declaram não haver conflitos de interesse.

\section{AUTOR PARA CORRESPONDÊNCIA}

\section{Marcela Filié Haddad}

Departamento de Odontologia Restauradora

Faculdade de Odontologia

Universidade Federal de Alfenas (UNIFAL-MG)

Rua Gabriel Monteiro da Silva, 700, Centro

37.130-000 Alfenas - MG, Brasil

Tel: (35) 3299-1464

Email: marcela.haddad@unifal-mg.edu.br

Submetido em 14/12/2020

Aceito em 16/12/2020 